\title{
Progression and Resolution of COVID-19 Pneumonia on Chest Radiograph
}

\author{
Laiba Masood, Suraya Bano Zafar, Madiha Saeed Wahla, Salma Gul, Samina Akhtar and Atif Iqbal Rana \\ Department of Radiology, Shifa International Hospital, Islamabad, Pakistan
}

\begin{abstract}
Objective: To evaluate the temporal changes on serial chest radiographs (CXRs)of hospitalised COVID-19 positive patients till their outcome(discharge/death); to determine the severity of CXR score and its correlation with clinical outcome (hospital stay, chest intubation and mortality).

Study Design: Descriptive study.

Place and Duration of Study: Shifa International Hospital (SIH), Islamabad from March to June 2020.

Methodology: After IRB approval, 112 patients were consecutively enrolled, having laboratory-confirmed SARS-CoV-2 and hospitalised in SIH. Patients' demographics and clinical data were retrieved from Radiology Information System (RIS). Chest radiographs (CXR) were retrieved from picture archive and communication system (PACS). CXR severity scoring was determined by three radiologists, and results were analysed.

Results: Lung opacities (98.2\%), involvement of both lungs (96.4\%), both peripheral and central region involvement $(62.5 \%)$ and upper/mid/lower zone distribution (61.6\%) were the most frequent findings. Males affected more than females with a mean age of $58.9 \pm 13.1$ years. Zonal involvement, density and extent of opacities peaked on 10-13 ${ }^{\text {th }}$ day of illness. In the last CXR, opacities showed decrease in extent as well as density, reduction in zonal involvement, and few having mixed interstitial thickening/fibrosis. One hundred and five out of 112 (93.8\%) patients had residual radiographic abnormalities on discharge.

Conclusion: Serial chest radiography can be used to monitor disease progression and temporal changes after initial HRCT. Patients who have CXR severity score of 4 or more at the time of admission, is a red flag for prolonged hospital stay and possible intubation. Severity of CXR findings peaked at 10-13 days. It is recommended to repeat CXRs every $3-4^{\text {th }}$ day during hospital stay. Majority of the patients has residual radiographic abnormality on discharge.
\end{abstract}

Key Words: COVID-19, Radiography, Thoracic, Pandemic, Chest X-ray.

How to cite this article: Masood L, Zafar SB, Wahla MS, Gul S, Akhtar S, Rana Al. Progression and Resolution of COVID-19 Pneumonia on Chest Radiograph. J Coll Physicians Surg Pak 2021; 31(03):258-261.

\section{INTRODUCTION}

Novel coronavirus 2019 (SARS-CoV-2 virus) is a highly contagious member of coronaviruses family. A large number of published articles have put emphasis on radiological findings of coronavirus disease (COVID -19) on chest high-resolution computed tomography (HRCT). ${ }^{1,2}$ However, problems related to infection-control, while transporting patients to CT suites, CT room disinfection, financial burden due to cost of CT and lack of accessibility to $\mathrm{CT}$ imaging in developing countries/remote areas, chest radiography (CXR) is the next most appropriate and commonly employed modality for diagnosis and review of lung pathologies.

Correspondence to: Dr. Laiba Masood, Department of Radiology, Shifa International Hospital, Islamabad,

Pakistan

E-mail: laibamasood66@gmail.com

Received: October 16, 2020; Revised: December 17, 2020;

Accepted: February 24, 2021

DOI: https://doi.org/10.29271/jcpsp.2021.03.258
It is also useful in detection of COVID where there is limited access to real-time reverse-transcriptase polymerase chain reaction (RT-PCR) COVID testing. ${ }^{3,4}$ Limited data is available regarding Pakistani patients where the virus has behaved differently, regarding severity and outcome, compared to many other parts of the world.

The aim of this study is to elaborate usefulness of CXR in current pandemic by evaluation of temporal changes on serial CXRs of hospitalised COVID-19 patients till their outcome (discharge / death), and severity of CXR score and its correlation with clinical status (hospital stay, chest intubation, and mortality).

\section{METHODOLOGY}

This descriptive study was performed retrospectively from March to June 2020, after Institutional Review Board's approval. Demographic details, information regarding day of onset of symptoms, and total hospital stay were drawn from Radiology Information System (RIS). Inclusion criteria was all admitted patients regardless of age or gender with positive SARS-CoV-2 RT-PCR result under at least three or more CXRs, performed during hospital stay. Patients not fulfilling these criteria were excluded. 
All CXRs were performed as postero-anterior (PA) or antero-posterior (AP) projection, following guidelines. ${ }^{4,5}$ Majority of radiographs were obtained in AP projection using portableX-ray units in the dedicated COVID units. ${ }^{6,7}$ Three radiologists having 5 to 15 years' experience reviewed the CXRs from PACS and compiled the data. Radiographic features including opacities, consolidations, interstitial thickening, and fibrosis were categorised by glossary of terms as proposed by Radiological Society of North America (RSNA) for COVID-19 CXR reporting. ${ }^{5}$

Pattern of the pulmonary findings were classified into (i) peripheral predominance, central prevalence, or both; (ii) right, left, or bilateral lung involvement; and (iii) upper, mid and lower zones (as described by Toussie et al.). ${ }^{1}$ Complications, such as pleural effusion, intubation, pneumothorax, and pneumomediastinum were also recorded. Hospital stay was labeled as prolonged if $>10$ days. $^{1}$

Opacity in each zone was scored by a CXR severity score as described by Toussie et al. and Borghesiet al. ${ }^{1,2}$, with 0 being minimum and 6 being maximum score. Each lung zone was assigned a number with a total of six lung zones: three on right upper, middle, and lower; similarly, three on left upper, middle, and lower zone, respectively. ${ }^{2}$ Mid zone was taken as area between two horizontal lines drawn from superior and inferior hilar markings. The lower zone between inferior hilar horn till costophrenic sulcus and the upper zone as the lung from superior hilarhorn upwards till the apices. ${ }^{2}$

Central distribution of lung opacities was defined as area within $2 \mathrm{~cm}$ from the lobar bronchial structures as far as perceivable. Peripheral zone was defined as the residual lung parenchyma present between the central area and the pleura. ${ }^{1-3}$

Virological testing was performed using Cobas 6800 system for the detection of SARS-CoV- 2RNA in nasopharyngeal swab samples collected in UTM-RT or BD UVT viral transport systems, followed by selective PCR amplification and detection of target nucleicacid. ${ }^{8}$

Statistical analysis was performed with IBM SPSS software version 21. Descriptive statistics were calculated for qualitative and quantitative variables, such as frequencies, percentages and mean \pm standard division (S.D). Likelihood ratio test of significance was used to determine association between CXR severity score and outcomes of interest. A $p$-value less than 0.05 was considered significant.

\section{RESULTS}

A total of 112 patients, 85 males (75.9\%) and 27 females (24.1\%), with mean age of 59.3 years \pm 12.6 (from 15 -90 years) were enrolled. Serial CXRs of all these patients were evaluated. Emphasis was placed on radiographs performed on admission, peak day of findings and at discharge/last day of hospitalisation.

A total of 846 CXRs were performed with an average $9.6 \pm 5.4$ CXRs for each patient during the hospital stay (range $3-33$ ). All 112 patients demonstrated CXR abnormalities during their hospital stay. One hundred and eleven patients had abnormal baseline CXR (Table I). Forty-two (37.5\%) of the admitted patients had a baseline severity score of 6.Bilateral mid and lower zone involvement with CXR severity score of 4 in 33 (29.5\%) patients, followed by score of 5 in $18(16.1 \%)$, a score of 3 in $8(7.1 \%)$ and CXR severity score of 2 with bilateral lower zone involvement in $6(5.4 \%)$, and unilateral lung involvement in 2 $(1.7 \%)$ patients. Both peripheral and central distribution of opacities $(70 / 112,62.5 \%)$, mid and lower zonal involvement $(111 / 112$, $99.1 \%)$, and bilateral lung involvement (108/112, 96.4\%) were commonest findings. On peak day of findings 106 patients demonstrated a CXR severity score of 4 or more (Figure 1 ).

Table I: Frequency of CXR severity score in 3 different points in time, ( $n=$ No. of patients).

\begin{tabular}{|l|c|c|c|}
\hline CXR severity score & $\begin{array}{c}\text { Day of } \\
\text { admission } \\
\%(\mathbf{n})\end{array}$ & $\begin{array}{c}\text { Peak day of } \\
\text { findings } \\
\%(\mathbf{n})\end{array}$ & $\begin{array}{c}\text { Day of } \\
\text { discharge } \\
\%(\mathbf{n})\end{array}$ \\
\hline Clear lungs - CXR Score 0 & $0.9(1)$ & $0(0)$ & $7.1(8)$ \\
\hline $\begin{array}{l}\text { Involvement of one zone - } \\
\text { CXR Score 1 }\end{array}$ & $0.9(1)$ & $0(0)$ & $0.9(1)$ \\
\hline $\begin{array}{l}\text { Involvement of two zones - } \\
\text { CXR Score 2 }\end{array}$ & $6.3(7)$ & $1.7(2)$ & $10.7(12)$ \\
\hline $\begin{array}{l}\text { Involvement of three zones - } \\
\text { CXR Score 3 }\end{array}$ & $6.3(7)$ & $3.6(4)$ & $2.7(3)$ \\
\hline $\begin{array}{l}\text { Involvement of four zones - } \\
\text { CXR Score 4 }\end{array}$ & $32.1(36)$ & $17.9(20)$ & $33.9(38)$ \\
\hline $\begin{array}{l}\text { Involvement of five zones - } \\
\text { CXR Score 5 }\end{array}$ & $16.0(18)$ & $15.2(17)$ & $9.8(11)$ \\
\hline $\begin{array}{l}\text { Involvement of six zones - } \\
\text { CXR Score 6 }\end{array}$ & $37.5(42)$ & $61.6(69)$ & $34.8(39)$ \\
\hline
\end{tabular}

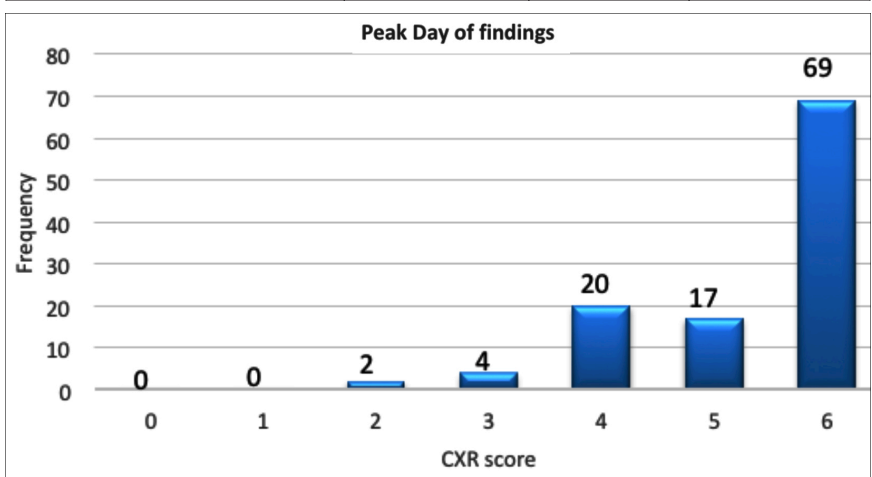

Figure 1: Frequency of radiograph chest severity score on peak day of findings with maximum number of patients having mean severity score of 6 .

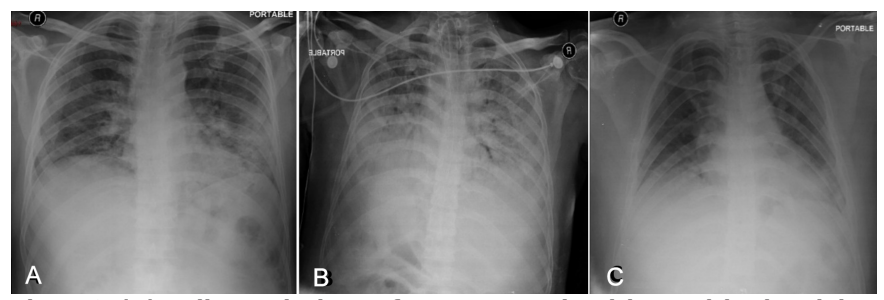

Figure2: (A)Radiograph chest of a 65-year male with opacities involving bilateral lower and mid zones in peripheral distribution and CXR severity score of 4 on the day of admission. (B) Peak day 12 findings with CXR severity score of 6 and interval intubation. (C) CXR at discharge showing interval improvement with severity score reduced to 2 and removal of ETT.

Ninety-six, out of 112 (85.7\%) admitted patients, showed interval worsening of CXR findings. One patient who had normal CXR on admission, also developed lung opacities on follow-up radiographs. Severity of CXR findings peaked at 10-13 days with 
(mean $11.0 \pm 3.3$ median day $11^{\text {th }}$ ) from the day of symptom onset, at which the median CXR severity score was 6. Out of 112, $91(81.3 \%)$ patients showed significant improvement in their CXR performed on last day of hospital admission, out of which 72 (79.1\%) showed significant reduction in the density of opacities as well as number of zonal involvement, while 11 (12.1\%) cases demonstrated interstitial thickening/fibrosis pattern and 8 $(8.8 \%)$ cases showed clear lungs.

Thirty-nine $(34.8 \%)$ patients were intubated with concurrent development of respiratory failure, pneumothorax in 4 (3.6\%), and pneumomediastinum in $3(2.7 \%)$. Five $(4.5 \%)$ patients had to be put on bilevel/continuous positive airway pressure (BiPAP/CPAP).Acute stroke was seen in $2(1.7 \%)$, pneumonia/sepsis in 6 (5.4\%), acute kidney injury in $8(7.1 \%)$, pleural effusion in $4(3.6 \%)$ and pulmonary/cardiogenic edema in 12 (10.7\%) patients.

Eighty patients (71.4\%) were discharged after a mean hospitalisation time span of 12 days \pm SD 6.6 (range 3-42 days). Ten patients $(8.9 \%)$ were discharged on request after a mean hospitalisation period of 5 days \pm SD of 2 , due to personal reasons. Twenty-two (19.6\%) patients unfortunately expired. Eighty (71.4\%) patients had prolonged hospital stay i.e. more than 10 days; out of which,24 (30.0\%) had a CXR severity score of 4 or more at the time of hospital admission ( $p$-value $=0.029)$. While CXR severity score of 4 or more was also associated with risk of intubation ( $p$-value $=0.015$ ). Correlation between high CXR severity score and patient mortality could not be established ( $p$ value $=0.078$ ), perhaps this could be attributed to a shorter samplesize.

\section{DISCUSSION}

Temporal changes were analysed on serial chest radiography of COVID positive admitted patients, which has not been explored so far in previously published studies. ${ }^{8-10}$ Serial CXRs of these patients were reviewed during hospital stay at different points of time, providing reliable data on thetemporal changes of radiographic pattern with emphasis on CXRs at the time of admission, peak day of findings, and on last day of hospital stay (Figure 2).

In the study by Toussie et al., CXR severity of 3 or more was independent predictor of intubation, while CXR severity of 2 or more required hospital admission. ${ }^{1}$ In the present study, pulmonary opacities in 4 zones or more were liable to require hospital admission, prolonged hospital stay, and chest intubation. Frequently reported CT findings of bilateral lung involvement, peripheral distribution, mid and lower zone predominance can also be appreciated on CXR. ${ }^{1,2,9,11-13}$

On the day of presentation, bilateral mid and lower zone peripheral lung opacities were the main finding on the CXRs. ${ }^{2,14,15}$ On the peak day of findings, the lung opacities gradually increased in extent and density with consolidative changes becoming the prevalent pattern as described. ${ }^{16,17}$ On the last day of hospital stay, a noticeable improvement in the density of opacities and total zonal involvement were observed with predominance of interstitial thickening and fibrosis.
In short, most of the patients showed the maximum lung changes on CXR at 10-13 days after the onset of symptoms. This is in concordance with findings described by Liu etal. and Pan et al. ${ }^{12}$

A short plateau phase in findings, followed by improvement, was identified in CXRatapproximately 15 days afterinitialsymptoms. ${ }^{18}$ Therefore, there is no need to repeat daily CXRs, unless under specific clinical demands, or if major intervention, such as intubation, venous access etc. are required. ${ }^{19}$

This study has few limitations. Firstly, some patients could not be followed up to their final outcome, since they were discharged once their symptoms improved. Secondly, in portable CXRs, superimposed findings, such as pulmonary edema and technical factors, limited the evaluation. ${ }^{1,2}$ Thirdly, as a tertiary care hospital, most of the patients had moderate to severe disease; and hence, this study does not give a true picture of temporal changes in mild COVID pneumonia.

CXR findings in patients with COVID-19 pneumonia can be utilised in disease-monitoring and follow-up of patients, who are to be hospitalised, after the initial screening/diagnostic HRCT. This will decrease the added financial burden and radiation dosage associated with repeat CT scans as well as reduce the viral loading in the CT suites, exposure to hospital staff and spread of virus in general hospital corridors.

\section{CONCLUSION}

Patients, who have CXR severity score of 4 or more at the time of admission, are red flags for prolonged hospital stay and possible intubation. Severity of CXR findings peaked at 10-13 days, and the authors recommend for CXRs to be repeated every $3-4^{\text {th }}$ day during hospital stay.

\section{ETHICALAPPROVAL:}

Ethical approval of this study was obtained from Shifa International Hospital Institution Review Board and Ethics Committee prior to initiation this research study.

\section{PATIENTS' CONSENT:}

As the study was designed retrospectively, data was collected from clinical archives after ethical approval.

\section{CONFLICT OF INTEREST:}

The authors declare no conflict of interest.

\section{AUTHORS' CONTRIBUTION:}

LM: Drafting manuscript, data collection, and interpretation of data.

SBZ, MSW: Drafting manuscript, data collection, and critical revision.

SG: Critical revision, final approval of version to be published.

SA: Data collection, critical revision, final approval.

AIR: Critical revision, final approval of version to be published.

\section{REFERENCES}

1. Toussie D, Voutsinas N, Finkelstein M, Cedillo MA, Manna S, Maron SZ, et al. Clinical and chest radiography features 
determine patient outcomes in young and middle age adults with COVID-19. Radiology 2020; 297(1):E197-E206. doi: 10.1148/radiol.2020201754.

2. Borghesi A, Maroldi R. COVID-19 outbreak in Italy: Experimental chest X-ray scoring system for quantifying and monitoring disease progression. Radiol Med 2020; 125(5):509-13. doi: 10.1007/s11547-020-01200-3.

3. Litmanovich DE, Chung M, Kicska G. Review of chest radiograph findings of COVID-19 Pneumonia and Suggested Reporting Language. J Thoracic Imaging 2020; doi: 10. 1097/RTI.0000000000000541.

4. Durrani M, ul Haq I, Kalsoom U, Yousaf A. Chest X-rays findings in COVID 19 patients at a university teaching hospital-A descriptive study. Pak J Med Sci 2020; 36 (COVID19-S4):S22-26. doi: 10.12669/pjms.36.COVID19S4.2778.

5. RSNA The Chest Radiograph in the COVID-19 Pandemic: Role, Standardized Reporting, \& Correlation [video on the internet]. 2020 April 14. Available from: http://www. youtube.com/watch?v=aSxOcyRd-bU.

6. Sarkodie BD, Osei-Poku K, Brakohiapa E. Diagnosing COVID-19 from Chest X-ray in Resource Limited Environment-Case Report. Med Case Rep 2020; 6(1):135.

7. Ng MY, Lee EY, Yang J, Yang F, Li X, Wang $\mathrm{H}$, et al. Imaging profile of the COVID-19 infection: Radiologic findings and literature review. Radiology: Cardiothoracic Imaging 2020; 2(1):e200034.

8. US Food and drug administration. Coronavirus testing basics. CLIA(Clinical Labortary Improvement Amendments. 2020 [updated 07/16/ 2020; cited 08/27/2020]. Available from :

http://www.fda.gov/consumers/consumer-updates/coronavi rus-testing-basics.

9. Pan F, Ye T, Sun P, Gui S, Liang B, Li L, et al. Time course of lung changes on chest CT during recovery from 2019 novel coronavirus (COVID-19) pneumonia. Radiology 2020; 295(3):715-21.doi: 10.1148/radiol.2020200370.

10. Liu X, Zhou H, Zhou Y, Wu X, Zhao Y, Lu Y, et al. Temporal radiographic changes in COVID-19 patients: Relationship to disease severity and viral clearance.Sci Rep 2020; 10(1):10263. doi: 10.1038/s41598-020-66895-w.
11. Zhang $Y$, Liu $Y$, Gong $H$, Wu L. Quantitative lung lesion features and temporal changes on chest CT in patients with common and severe SARS-CoV-2 pneumonia. Plos One2020;15(7):e0236858.doi: 10.1371/ journal.pone. 0236858 .

12. Wang $\mathrm{Y}$, Dong $\mathrm{C}, \mathrm{Hu}$ Y, Li C, Ren Q, Zhang X, et al. Temporal changes of CT findings in 90 patients with COVID-19 pneumonia: A longitudinal study. Radiology 2020; 296(2):E-55-64.doi: 10.1148/radiol.2020200843.

13. Vancheri SG, Savietto G, Ballati F, Maggi A, Canino C, Bortolotto C, et al. Radiographic findings in 240 patients with COVID-19 pneumonia: time-dependence after the onset of symptoms. Eur Radiol 2020; 30(11):6161-69.doi: 10.1007/s00330-020-06967-7.

14. Stogiannos N, Fotopoulos D, Woznitza N, Malamateniou C. Coronavirus disease 2019 (COVID-19) in the radiology department: What radiographers need to know. Radiography2020; 26(3):254-63. doi: 10.1016/j.radi. 2020.05.012.

15. Shi H, Han X, Jiang N, Cao Y, Alwalid O, Gu J, et al. Radiological findings from 81 patients with COVID-19 pneumonia in Wuhan, China: A descriptive study. Lancet Infect Dis 2020; 20(4):425-34. doi: 10.1016/S14733099(20)30086-4.

16. Rubin GD, Ryerson CJ, Haramati LB, Sverzellati N, Kanne JP, Raoof $\mathrm{S}$, et al. The role of chest imaging in patient management during the COVID-19 pandemic: A multinational consensus statement from the Fleischner Society. Chest 2020; 158(1):106-116. doi: 10.1016/j. chest.2020.04.003.

17. Sohail S. Radiology of COVID-19-imaging the pulmonary damage. J Pak Med Assoc2020; 70(5):S60-3.doi: 10. 5455/JPMA.21.

18. Jacobi A, Chung M, Bernheim A, Eber C. Portable chest Xray in coronavirus disease-19 (COVID-19): A pictorial review. Clin Imaging 2020; 64:35-42. doi: 10.1016/ j.clinimag.2020.04.001.

19. Wei J, Xu H, Xiong J, Shen Q, Fan B, Ye C, et al. 2019 novel coronavirus (COVID-19) pneumonia: Serial computed tomography findings. Korean J Radiol 2020; 21(4):501-4. doi: 10.3348/kjr.2020.0112. 